\title{
Traditional Chinese Medicine for Senile Dementia
}

\author{
Zhihong Lin, ${ }^{1}$ Jie Gu, ${ }^{1}$ Jin Xiu, ${ }^{1}$ Tingyan Mi, ${ }^{1}$ Jie Dong, ${ }^{1}$ and Jyoti Kumar Tiwari ${ }^{2}$ \\ ${ }^{1}$ Naturals and Bioscience, Unilever ReD Shanghai, Shanghai 200233, China \\ ${ }^{2}$ Molecular Aspects of Health, Unilever R\&D Vlaardingen, Vlaardingen 3130 AC, The Netherlands
}

Correspondence should be addressed to Zhihong Lin, nercmtcm@hotmail.com

Received 12 January 2011; Accepted 9 May 2011

Academic Editor: Virginia S. Martino

Copyright ( $) 2012$ Zhihong Lin et al. This is an open access article distributed under the Creative Commons Attribution License, which permits unrestricted use, distribution, and reproduction in any medium, provided the original work is properly cited.

Traditional Chinese Medicine (TCM) has a 3000 years' history of human use. A literature survey addressing traditional evidence from human studies was done, with key result that top 10 TCM herb ingredients including Poria cocos, Radix polygalae, Radix glycyrrhizae, Radix angelica sinensis, and Radix rehmanniae were prioritized for highest potential benefit to dementia intervention, related to the highest frequency of use in 236 formulae collected from 29 ancient Pharmacopoeias, ancient formula books, or historical archives on ancient renowned TCM doctors, over the past 10 centuries. Based on the history of use, there was strong clinical support that Radix polygalae is memory improving. Pharmacological investigation also indicated that all the five ingredients mentioned above can elicit memory-improving effects in vivo and in vitro via multiple mechanisms of action, covering estrogenlike, cholinergic, antioxidant, anti-inflammatory, antiapoptotic, neurogenetic, and anti-A $\beta$ activities. Furthermore, 11 active principles were identified, including sinapic acid, tenuifolin, isoliquiritigenin, liquiritigenin, glabridin, ferulic acid, Z-ligustilide, $\mathrm{N}$-methyl-beta-carboline-3-carboxamide, coniferyl ferulate and 11-angeloylsenkyunolide $\mathrm{F}$, and catalpol. It can be concluded that TCM has a potential for complementary and alternative role in treating senile dementia. The scientific evidence is being continuously mined to back up the traditional medical wisdom.

\section{Introduction}

Cognitive impairment or dementia in elderly is associated with many disorders [1]. Alzheimer's disease (AD) is the principal type of dementia and represents about $70 \%$ of the dementia patients.

The pathologic hallmarks of $\mathrm{AD}$ are senile plaques, neurofibrillary tangles, dystrophic neurites, and neuronal loss. The development of $\mathrm{AD}$ may be due to the improper biochemical processing of amyloid precursor protein (APP) leading to subsequent accumulation of $\beta$-amyloid $(A \beta)$. The amyloid and tangle cascade hypothesis is the dominant explanation for the pathogenesis of $\mathrm{AD}$ [2]. Other relevant factors, including cholinergic dysfunction [3], neuroinflammation $[4,5]$, oxidative stress [6], and disturbance of neuronal plasticity [7], age-related loss of sex hormones $[8,9]$, are important and contribute to the understanding of $\mathrm{AD}$ pathology.

The 2nd most common form of dementia is vascular dementia (VD) or multi-infarct dementia, which accounts for about $15 \%$ of dementia cases $[10,11]$. VD may fol- low after a succession of acute cerebrovascular events or, less commonly, a single major stroke. The compromised cerebrovascular circulation causes ischemia that leads to damage of the brain structure, for example, formation of white matter lesions or silent brain infarctions. VD is often related to the loss of fine motor control besides memory impairment.

Currently, there is no effective treatment for $\mathrm{AD}$, although many treatment strategies exist [12]. Clinically, cholinesterase inhibitors (ChEIs) and N-methyl-D-aspartate (NMDA) receptor antagonists are first-line pharmacotherapy for mild-to-moderate $\mathrm{AD}$, with high nonresponse rate $50-75 \%$ [13].

Lots of folk plants in traditional medicine are being used in age-related brain disorders for improvement of memory and cognitive function [14-16]. In China, a number of herb ingredients known from Traditional Chinese Medicine (TCM) have a long history of use for mental health. In this study, we exploited the empirically driven TCM lore and surveyed scientific data to back up the cognitive benefits, claimed by TCM. 


\section{Ancient Records on TCM for Cognitive Decline}

The term "senile dementia" refers to a clinical syndrome seen in the elderly characterized by impairment of memory and cognition. So in a search of the ancient literature of TCM, the etiology, pathogenesis, and treatment for "dementia or amnesia" have been used for the survey in detail.

\subsection{Etiology and Pathogenesis}

2.1.1. Deficiency of Energy. Deficiency of energy is similar to "Q $i$ " deficiency in TCM. According TCM lore Qi is the essential substance that makes up the body and maintains various physiological activities, similar to flow of energy in the body. The energy is mainly from the kidney, heart, and spleen, especially from the kidney. In TCM, the energy from the kidney is called kidney essence which can produce marrow including cerebral marrow, spinal cord, and bone marrow. The cerebral marrow can nourish the brain and maintain the physiological functions of the brain. If the kidney essence is insufficient, the production of cerebral marrow will be reduced, leading to various symptoms, such as headache, dizziness, amnesia, and retard response [17].

2.1.2. Blood Stasis. Normally the blood is pumped by the heart to flow in the vessels. If blood circulation is stagnated or slowed down by certain factors such as cold, emotional disorder, aging, consumptive disease, and overstrain, it will result in retention of blood flow in the vessels or organs, a pathological condition named blood stasis. The cognitive function will decline, due to long-term global hypo-perfusion in cerebral blood flow or acute focal stroke in memory-related cerebral parenchyma [17].

2.1.3. Toxin. As the function of internal organs in the elderly decline, the balance between host defense and external toxins in the body is disrupted. Pathological or physiological products occur and form toxin including waste of "water" and "endogenous fire", which result from the poor digestion, accumulates into phlegm and retention of fluid, and caused by mental disorder, attack from pathological factors, and imbalance within the body, respectively. If such toxins can not be eliminated quickly the blood circulation and mental acuity will be affected, eventually contributing to the onset of dementia.

2.2. Therapy of TCM. TCM has a long history for preventing and treating cognitive decline. Although $\mathrm{AD}$ is a modern disease entity and has no direct analogue in the ancient Chinese medicine literature, disorders of memory and cognitive deficit are referred to throughout the classical literature. For example, in Sheng Nong Ben Cao Jing (Han dynasty, 1-2 century), the earliest pharmacopeia existing on materia medica in China, some TCM ingredients such as Yuan Zhi (Thinleaf milkwort), Ren Shen (Ginseng), Huang Lian (Golden thread), and Long Yan (Longan) were recorded to ameliorate the decline of people's memory.
In this study, 27 ancient TCM books were selected, which could be divided into 3 types, namely, Pharmacopoeias, formulae monographs and renowned TCM doctor's case studies.

A database was established to determine the frequency of herbs in these documents. Totally 236 formulae for improving cognitive function were identified among 27 books mentioned above (Table 1); 139 herbs were gathered from those 236 formulae and 10 TCM herbs were prioritized due to the highest frequency over 50 times (Table 2).

According to specification documented in Chinese Pharmacopeia [18], (i) Poria cocos is a diuretic with capacity to invigorate spleen function and calm the mind. Clinically, it is applicable for memory decline due to spleen deficiency and phlegm blockage; (ii) Radix polygalae is able to anchor the mind and eliminate the phlegm, and indicated in forgetfulness and insomnia; (iii) Radix glycyrrhizae is a $q i$ tonic to invigorate the stomach and spleen, resolve phlegm, and clear away heat and toxin; (iv) Radix Angelica sinensis, as a vital blood tonic and antithrombotic agent, is especially used to treat stroke and poststroke vascular dementia induced by blood stasis; (v) Radix rehmanniae is another tonic used to reinforce kidney essence and marrow. Because of functionality to invigorate the energy, activate blood circulation, or eliminate the toxin, these herbs can be prescribed along or combined to exhibit a good therapeutic effect for senile dementia, for example, Zhi Ling Tang [19].

\section{Evidence-Based Efficacy of TCM Herbs on Cognitive Decline}

3.1. Poria cocos. Poria cocos (Chinese name: Fu Ling) is the dried sclerotium of the fungus, Poria cocos (Schw.) Wolf (Fam. Polyporaceae).

3.1.1. Functionality/Efficacy. There is suggestive evidence that $P$. cocos is memory improving regardless of absence of available clinical reports. Pharmacological research exhibited that the water extract of $P$. cocos enhanced hippocampal long-term potentiation (LTP) and improved scopolamineinduced spatial memory impairment in rats $([20,21]$, Table 3).

3.1.2. Mechanism of Action. Its cognitive action has been ascribed to slight cholinesterase (ChE) or acetylcholinesterase (AChE) inhibition and bidirectional regulation on cytosolic free calcium ([22-24], Table 3).

3.1.3. Active Principles. The responsible actives for the cognitive benefits are unclear for the time being. Triterpene acids and polysaccharides are principal constituents of $P$. cocos, responsible for diverse bioactivities, including antitumor, anti-inflammatory, nematicidal, antioxidant, antirejection, antiemetic effects, as inhibitors against DNA topoisomerases, phospholipase A2. Besides, lecithin and choline present in the fungus are beneficially nutritional substance [25-29].

3.2. Radix polygalae. Radix polygalae is the root Polygala tenuifolia Willd. or P. sibirica L. (Fam. Polygalaceae), used 
TABLE 1: TCM formulae selected from ancient Chinese documents.

\begin{tabular}{|c|c|c|c|}
\hline Classification & Book name & Dynasty & $\begin{array}{c}\text { Formulae } \\
\text { amount }\end{array}$ \\
\hline \multirow{6}{*}{ Pharmacopoeia } & Sheng Ji Zhong Lu & Song ( $10-13$ century) & 45 \\
\hline & Tai Ping Hui Min He Ji Ju Fang & Song (10-13 century) & 2 \\
\hline & Tai Ping Sheng Hui Fang & Song (10-13 century) & 2 \\
\hline & Pu Ji Fang & Ming (14-17 century) & 2 \\
\hline & Yi Fang Lei Ju & Ming (14-17 century) & 2 \\
\hline & Yi Zong Jin Jian & Qing (17-19 century) & 9 \\
\hline \multirow{11}{*}{$\begin{array}{l}\text { Formulae } \\
\text { monographs }\end{array}$} & Zhou Hou Fang & Jin (3-4 century) & 1 \\
\hline & Qian Jin Yao Fang & Tang (7-10 century) & 3 \\
\hline & Ren Zhai Zhi Zhi Fang Lun & Song (10-13 century) & 3 \\
\hline & Fu Ren Da Quan Liang Fang & Song (10-13 century) & 1 \\
\hline & Shi Zhai Bai Yi Xuan Fang & Song (10-13 century) & 5 \\
\hline & Shi Yi De Jiu Fang & Yuan (13-14 century) & 4 \\
\hline & Qi Xiao Liang Fang & Ming (14-17 century) & 29 \\
\hline & Gu Jin Yi Jian & Ming (14-17 century) & 1 \\
\hline & She Sheng Zhong Miao Fang & Ming (14-17 century) & 1 \\
\hline & Zheng Zhi Bao Jian & Qing (17-19 century) & 1 \\
\hline & Ji Yan Liang Fang & Qing (17-19 century) & 4 \\
\hline \multirow[t]{10}{*}{ Medical edition } & Yan Yonghe's medical edition & Song (10-13 century) & 13 \\
\hline & Chen Wuze's medical edition & Song (10-13 century) & 9 \\
\hline & Dan Xi Xin Fa & Yuan (13-14 century) & 4 \\
\hline & Shou Shi Bao Yuan & Ming (14-17 century) & 21 \\
\hline & Jing Yue Quan Shu & Ming (14-17 century) & 21 \\
\hline & Zheng Ti Lei Yao & Ming (14-17 century) & 2 \\
\hline & Lei Zheng Zhi Chai & Qing (17-19 century) & 16 \\
\hline & Bian Zheng Lu & Qing (17-19 century) & 16 \\
\hline & Zha Bing Yuan Liu Xi Zhu & Qing (17-19 century) & 2 \\
\hline & Yi Xue Zhong Zhong Can Xi Lu & Modern (20 century) & 7 \\
\hline Sum & & & 236 \\
\hline
\end{tabular}

TABLE 2: Top 10 memory-improving TCM herbs.

\begin{tabular}{|c|c|c|c|c|c|}
\hline Chi name & English name & Latin name & Part & Plant & Frequency \\
\hline Fu Ling & Poria & Poria cocos & Sclerotium & Poria $\operatorname{cocos}$ (Schw.) Wolf & 182 \\
\hline Ren Shen & Ginseng & Radix et rhizoma ginseng & Root, stem & Panax ginseng C. A. Mey. & 169 \\
\hline Yuan Zhi & Thinleaf milkwort & Radix polygalae & Root & $\begin{array}{l}\text { Polygala tenuifolia willd. } \\
\text { Polygala sibirica L. }\end{array}$ & 139 \\
\hline Gan Cao & Licorice & $\begin{array}{l}\text { Radix et rhizoma } \\
\text { glycyrrhizae }\end{array}$ & Root, stem & $\begin{array}{l}\text { Glycyrrhiza inflata Bat. } \\
\text { Glycyrrhiza uralensis Fisch. } \\
\text { Glycyrrhiza grabra L. }\end{array}$ & 100 \\
\hline Dang Gui & Chinese Angelica & Radix Angelica sinensis & Root & $\begin{array}{l}\text { Angelica sinensis (Oliv.) } \\
\text { Diels }\end{array}$ & 84 \\
\hline Shi Chang Pu & Grassleaf sweelflag rhizome & Rhizoma acori tatarinowii & Stem & Acorus tatarinowii Schott. & 80 \\
\hline Suan Zao Ren & Spina date seed & Semen ziziphi spinosae & Seed & $\begin{array}{l}\text { Ziziphus jujuba } \\
\text { Mill.var.spinosa. (Bunge) } \\
\text { Hu ex H.F. Chou }\end{array}$ & 79 \\
\hline Shu Di Huang & Prepared rehmannia root & Radix rehmanniae & Root & $\begin{array}{l}\text { Rehmannia glutinosa } \\
\text { Libosch. }\end{array}$ & 62 \\
\hline Mai Dong & Dwarf lilyturf tuber & Radix ophiopogonis & Root & $\begin{array}{l}\text { Ophiopogon japonicus (L.f.) } \\
\text { Ker-Gawl. }\end{array}$ & 62 \\
\hline Sheng Jiang & Fresh ginger & Rhizoma zingiberis & Stem & Zingiber officinale Rosc. & 53 \\
\hline
\end{tabular}

(Note: data are cited from Pharmacopoeia of PR China 2005). 
Table 3: Memory-improving and neuro-protective effects of Poria cocos.

\begin{tabular}{|c|c|c|c|c|c|}
\hline Test & Test materials/dose & Test model & Endpoints/biomarkers & Effects & Reference \\
\hline \multirow{4}{*}{ In vivo } & $\begin{array}{l}\text { Extracts } \\
20-100 \mathrm{mg} / \mathrm{kg}\end{array}$ & Scopolamine-treated rats & Eight-arm radial maze & Improve spatial memory & {$[20]$} \\
\hline & $\begin{array}{l}\text { Extracts } \\
250-500 \mathrm{mg} / \mathrm{kg}\end{array}$ & Innate rats & $\begin{array}{l}\text { Electro-physiology } \\
\text { Spike amplitude }\end{array}$ & Enhance hippocampal LTP & {$[21]$} \\
\hline & $\begin{array}{l}\text { Methanol extracts } \\
200 \mathrm{mg} / \mathrm{mL}\end{array}$ & Ellman ChE & ChE activity & Inhibit ChE by $27.8 \%$ & {$[22]$} \\
\hline & $\begin{array}{l}\text { Aqueous extracts } \\
0.2 \mathrm{mg} / \mathrm{mL}\end{array}$ & Innate ICR mice & AChE activity & Inhibit AChE by $13.9 \%$ & {$[23]$} \\
\hline In vitro & $\begin{array}{l}\text { Aqueous extracts } \\
31-250 \mu \mathrm{g} / \mathrm{mL}\end{array}$ & Brain neurons-neonatal rats & Cytosolic $\left[\mathrm{Ca}^{2+}\right] \mathrm{i}$ & Regulate bi-directly $\left[\mathrm{Ca}^{2+}\right] \mathrm{i}$ & {$[24]$} \\
\hline
\end{tabular}

Long-term potentiation (LTP); choline esterase (ChE); acetylcholinesterase (AChE).<smiles>COc1cc(/C=C/C(=O)O)cc(OC)c1O</smiles>

Sinapic acid

(a)

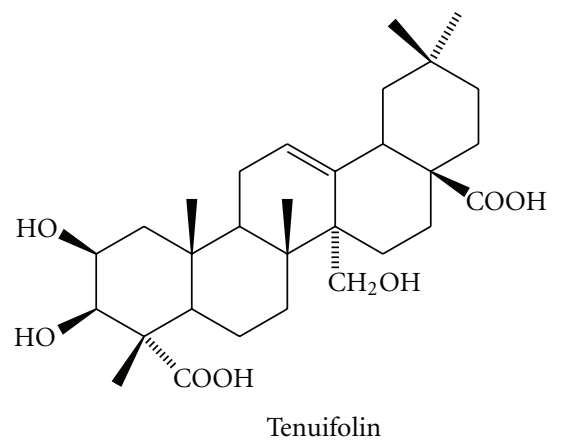

(b)

FIgURE 1: Chemical structures of sinapic acid and tenuifolin.

as a cardiotonic and cerebrotonic, sedative and tranquilliser, and for amnesia, neuritis, and insomnia [30, 31].

3.2.1. Functionality/Efficacy. There is strong support that thinleaf milkwort root is memory improving. BT-11, the extract of dried root of Radix polygalae, was developed in Korea as a functional diet with cognitive enhancing activity. Elderly with subjective memory impairment and mild cognitive impairment ascend with oral BT-11 at $300 \mathrm{mg} / \mathrm{d}$ for 4-8 weeks. Except for mild dyspepsia, no adverse events were reported $[32,33]$.

3.2.2. Mechanism of Action. A number of investigations also sustained that Radix polygalae extracts functioned to promote neuronal proliferation and neurite outgrowth in normal brain and improve memory impaired by scopolamine, stress, nucleus basalis magnocellularis-lesioning operation via a variety of molecular pathways, including increasing glucose utilization and inhibiting AChE activity. Besides nootropic effects, Radix polygalae extracts protected neurons against insults induced by NMDA, glutamate, and A $\beta$ ([3439], Table 4(a)). In addition, anti-inflammatory activity probably contributed to the cognitive and neuroprotective efficacy, as Radix polygalae extracts inhibited interleukin-1 (IL-1)-mediated tumour necrosis factor (TNF)- $\alpha$ secretion, and ethanol-induced IL-1 secretion by astrocytes [40, 41].
3.2.3. Active Principles. Phytochemically, Radix polygalae mainly contains a variety of active constituents, including saponins, xanthones, and acylated oligiosaccharides [42-44].

Saponins, especially tenuifolin isolated from tenuigenin might reinforce cognitive performance in aged and dysmnesia mice, via elevating levels of dopamine (DA) and norepinephrine (NE), and inhibiting AChE activity (Figure 1). Meanwhile, onjisaponin indicated cytoprotective activity in PC12 cells, exposed to serum deficiency or glutamate. In addition, tenuigenin facilitated memory in rats, damaged by A $\beta 1-40$ or ibotenic acid, via enhancing cholinergic function, or inhibiting $\mathrm{A} \beta$ secretion ([45-48], Table 4(b)).

Few phytochemical principles have been isolated and identified as CNS active components. Besides tenuifolin, sinapic acid [49], a common moiety of tenuifoliside B and 3, 6'-disinapoylsucrose, reversed memory deficit induced by scopolamine and basal forebrain lesion (Table 4(b), Figure 1).

3.3. Radix et Rhizoma Glycyrrhizae. Radix et rhizoma glycyrrhizae is the dried root and rhizome, generally derived from a different plant species, with similar properties, including Glycyrrhiza uralensis Fisch., G. inflata Bat., or G. glabra L. (Fam. Leguminosae).

3.3.1. Functionality/Efficacy. The extracts of Radix glycyrrhizae reversed the cognitive deficits induced by diazepam, 
TABle 4

(a) Memory-improving and neuro-protective effects of Radix polygalae

\begin{tabular}{|c|c|c|c|c|c|c|}
\hline Test & Test materials/dose & Test model & Endpoint/biomarkers & Effects & Mechanisms & Reference \\
\hline Clinic & $\begin{array}{l}\text { Extracts } \\
300 \mathrm{mg} / \mathrm{d}, 4 \mathrm{w}\end{array}$ & $\begin{array}{l}\text { Healthy Korean elderly } \\
\text { with subjective memory } \\
\text { impairment and mild } \\
\text { cognitive impairment } \\
\text { double-blind, } \\
\text { placebo-controlled, } \\
\text { randomized, parallel study }\end{array}$ & $\begin{array}{l}\text { Korean version of } \\
\text { California verbal } \\
\text { learning test } \\
\text { Self-ordered pointing } \\
\text { test }\end{array}$ & $\begin{array}{l}\text { Improve verbal } \\
\text { memory } \\
\text { No adverse events, } \\
\text { except mild } \\
\text { dyspepsia }\end{array}$ & N.A. & {$[32,33]$} \\
\hline \multirow{4}{*}{ In vivo } & $\begin{array}{l}\text { Extracts } \\
\text { i.p., } 2 \mathrm{mg} / \mathrm{kg}\end{array}$ & Innate rats & $\begin{array}{l}\text { Nestin/BrdU } \\
\text { Tuj1/BrdU }\end{array}$ & $\begin{array}{l}\text { Improve memory } \\
\text { Promote } \\
\text { neuro-genesis }\end{array}$ & $\begin{array}{l}\text { Promote } \\
\text { proliferation } \\
\text { Promote neurite } \\
\text { outgrowth }\end{array}$ & {$[34]$} \\
\hline & Extracts & Stress-treated rats & $\begin{array}{l}\text { Glucose utilization } \\
\text { Cell adhesion molecule }\end{array}$ & Improve memory & $\begin{array}{l}\text { Increase glucose } \\
\text { utilization } \\
\text { Increase total } \\
\text { NCAM }\end{array}$ & {$[35]$} \\
\hline & $\begin{array}{l}\text { Extracts } \\
2 \mathrm{~g} / \mathrm{kg}, 1-3 \mathrm{w}\end{array}$ & NBM-lesioning rats & $\begin{array}{l}\text { Neurological test } \\
\text { Step-through test }\end{array}$ & Improve memory & N.A. & {$[36]$} \\
\hline & $\begin{array}{l}\text { Extracts } \\
\text { i.p., } 10 \mathrm{mg} / \mathrm{kg}\end{array}$ & Scopolamine-treated rats & $\begin{array}{l}\text { Passive avoidance test } \\
\text { water maze test } \\
\text { AChE }\end{array}$ & Improve memory & Inhibit AChE & {$[36]$} \\
\hline \multirow{3}{*}{ In vitro } & $\begin{array}{l}\text { Extracts } \\
0.5-5 \mu \mathrm{g} / \mathrm{mL}\end{array}$ & $\begin{array}{l}\text { Rat primary neurons } \\
\text { exposed to Glutamate or } \\
\text { A } \beta\end{array}$ & Cell viability & Protect neurons & N.A. & [37] \\
\hline & $\begin{array}{l}\text { Extracts } \\
0.05-5 \mu \mathrm{g} / \mathrm{mL}\end{array}$ & $\begin{array}{l}\text { Rat cerebellar granule } \\
\text { neurons exposed to NMDA }\end{array}$ & $\begin{array}{l}\text { Glutamate release } \\
(\mathrm{Ca} 2+) \mathrm{i} / \mathrm{ROS}\end{array}$ & Protect neurons & N.A. & {$[38]$} \\
\hline & $\begin{array}{l}\text { Extracts } \\
0.1-100 \mu \mathrm{g} / \mathrm{mL}\end{array}$ & $\begin{array}{l}\text { Rat cortical neurons } \\
\text { exposed to } A \beta 25-35\end{array}$ & $\begin{array}{l}\text { Axonal length } \\
\text { Neuro-filament- } \\
\text { H/MAP-2 } \\
\text { Cell viability }\end{array}$ & $\begin{array}{l}\text { Activate axonal } \\
\text { extension Protect } \\
\text { neurons }\end{array}$ & N.A. & {$[39]$} \\
\hline
\end{tabular}

Acetylcholinesterase (AChE); bromodeoxyuridine (BrdU); microtubule-associated protein-2 (MAP-2); nucleus basalis magnocellularis (NBM); neural cell adhesion molecule (NCAM); N-methyl-D-aspartic acid (NMDA); reactive oxygen species (ROS); $\beta$ amyloid (A $\beta$ ); not available (N.A.); intraperitoneally (ip.).

(b) Memory-improving and neuro-protective effects of active components from Radix polygalae

\begin{tabular}{|c|c|c|c|c|c|c|}
\hline Test & Test materials/dose & Test model & Endpoints/biomarkers & Effects & Mechanisms & Reference \\
\hline \multirow{5}{*}{ In vivo } & $\begin{array}{l}\text { Sinapic acid } \\
10-100 \mathrm{mg} / \mathrm{kg}\end{array}$ & $\begin{array}{l}\text { Scopolamine-treated } \\
\text { rats }\end{array}$ & Radial maze test & Improve memory & N.A. & {$[42,43]$} \\
\hline & $\begin{array}{l}\text { Sinapic acid } \\
3-100 \mathrm{mg} / \mathrm{kg}, 1 \mathrm{~h}\end{array}$ & $\begin{array}{l}\text { Scopolamine-treat mice } \\
\text { Basal forebrain lesioning } \\
\text { mice }\end{array}$ & $\begin{array}{l}\text { Step-through test } \\
\text { Ach/ChAT }\end{array}$ & Improve memory & N.A. & {$[49]$} \\
\hline & $\begin{array}{l}\text { Tenuifolin } \\
20-80 \mathrm{mg} / \mathrm{kg}, 15 \mathrm{~d}\end{array}$ & $\begin{array}{l}\text { Aged mice Dysmnesia } \\
\text { mice }\end{array}$ & $\begin{array}{l}\text { Step-down test } \\
\text { Y maze trial } \\
\text { AChE,NE,DA,5-HT }\end{array}$ & Improve memory & $\begin{array}{l}\text { Increase } \mathrm{NE} \text { and } \\
\mathrm{DA} \\
\text { Inhibit } \mathrm{AChE}\end{array}$ & {$[45]$} \\
\hline & $\begin{array}{l}\text { Tenuigenin } \\
18.5-74 \mathrm{mg} / \mathrm{kg}\end{array}$ & $\begin{array}{l}\text { A } \beta 1-40 \text {-treated rats } \\
\text { ibotenic acid-treated rats }\end{array}$ & $\begin{array}{l}\text { Step-through test } \\
\text { AchE, ChAT }\end{array}$ & Improve memory & Cholinergic & {$[46]$} \\
\hline & $\begin{array}{l}\text { Acylated } \\
\text { oligosaccharides } \\
1-10 \mathrm{mg} / \mathrm{kg}\end{array}$ & $\begin{array}{l}\text { Scopolamine-treated } \\
\text { rats }\end{array}$ & Step-through test & Improve memory & Cholinergic & {$[44]$} \\
\hline \multirow{2}{*}{ In vitro } & $\begin{array}{l}\text { Tenuigenin } \\
1-4 \mu \mathrm{g} / \mathrm{mL}\end{array}$ & $\begin{array}{l}\text { APP-transfected } \\
\text { SH-SY5Y cells }\end{array}$ & $\begin{array}{l}\text { Fluorescence resonance } \\
\text { energy transfer }\end{array}$ & Inhibit $\mathrm{A} \beta$ secretion & Inhibit BACE1 & {$[47]$} \\
\hline & $\begin{array}{l}\text { Onjisaponin } \\
10 \mu \mathrm{M}\end{array}$ & $\begin{array}{l}\text { Serum deficiency or } \\
\text { glutamate-treated PC12 } \\
\text { cells }\end{array}$ & Cell survival & Protect PC 12 cells & N.A. & {$[48]$} \\
\hline
\end{tabular}

Acetylcholine (Ach); acetylcholinesterase (AChE); choline acetyltransferase (ChAT); 5-hydroxytryptamine (5-HT); dopamine (DA); norepinephrine (NE); beta-site APP cleaving enzyme (BACE); amyloid precursor protein (APP); $\beta$ amyloid (A $\beta$ ); not available (N.A.). 


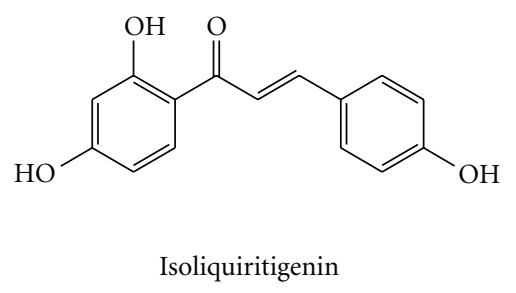

(a)

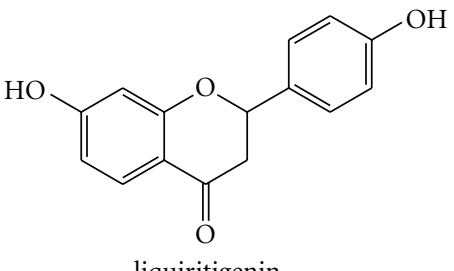

liquiritigenin

(b)

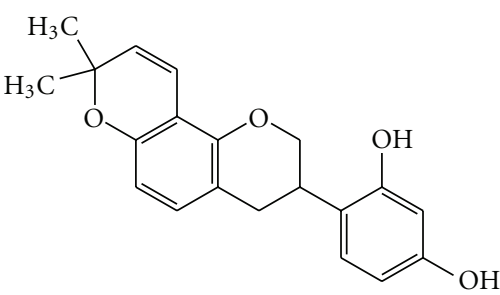

Glabridin

(c)

FIGURE 2: Chemical structures of isoliquiritigenin, liquiritigenin, and glabridin.

scopolamine, and beta-amyloid peptide $25-35$ in mice at doses of 75,150 , and $300 \mathrm{mg} / \mathrm{kg}$ per oral, or diet containing either 0.5 or $1 \%$ extract, through anti-AChE and antioxidant activities. In addition, roasted licorice extracts elicited neuroprotection against brain damage after transient forebrain ischemia in Mongolian gerbils, behind which antioxidant activity was also implicated, for example, maintaining superoxide dismutase (SOD) 1 level in hippocampal CA1 pyramidal cells ([50-54], Table 5).

3.3.2. Mechanism of Action and Active Principles. Radix glycyrrhizae contains glycyrrhizin, glycyrrhizic acid, glabridin and derivatives, glabrol, glabrene, $17 \beta$-hydroxysteroid dehydrogenase, glucoliquiritin apioside, prenyllicoflavone A, shinflavone, shinpterocarpin, 1-methoxyphaseollin, salicylic acid, and derivatives, as well as other saponins, flavonoid glycosides, and flavonoids.

Isoliquiritigenin, liquiritigenin, and glabridin have been identified from the Radix glycyrrhizae to be possible bioactive compounds ([55-58], Table 5, Figure 2).

(1) Isoliquiritigenin also has the protective potential against transient middle cerebral artery occlusioninduced focal cerebral ischemia in rats, at the doses of 5,10 , and $20 \mathrm{mg} / \mathrm{kg}$. Its protection may be attributed to amelioration of cerebral energy metabolism and antioxidant property.

(2) Liquiritigenin, a plant-derived highly selective estrogen receptor $\beta$ agonist has been identified to alleviate the cognitive recession in the elders.

(3) Glabridin appears to be an active isoflavone as it improved learning and memory in mice at 1,2 , and $4 \mathrm{mg} / \mathrm{kg}$, through targeting at ChE. Glabridin had a protective effect on cerebral ischemia injury, and neuron insult induced by staurosporine at 5 , $25 \mathrm{mg} / \mathrm{kg}$ (i.p). Its underlying mechanism is probably linked to antioxidant and antiapoptotic activity.

(4) Glabrene also could be beneficial to memory due to estrogen-like activities, like isoliquiritigenin, liquiritigenin, and glabridin [59-61].

3.4. Radix Angelica sinensis. Radix Angelica sinensis (Chinese: Danggui, Dong quai, Donggui; Korean Danggwi), is the dried root of Angelica sinensis (Oliv.) Diels (Umbelliferae).
3.4.1. Functionality/Efficacy. Behaviour test displayed that Radix Angelica sinensis extracts ameliorated scopolamine and cycloheximide, but not p-chloroamphetamine-induced amnesia at $1 \mathrm{~g} / \mathrm{kg}$ bw. In addition in vitro study showed that Radix Angelica sinensis extracts prevented the neurotoxicity induced by $\mathrm{A} \beta$ in Neuro $2 \mathrm{~A}$ cells, at the doses ranging $25-200 \mu \mathrm{g} / \mathrm{mL}$, through antioxidant pathway ([62, 63], Table 6(a)). Furthermore, estrogenic activity of Angelica sinensis will probably help alleviate peri- or postmenopausal symptoms including cognitive decline in women $[64,65]$.

\subsubsection{Mechanism of Action and Active Principles}

(1) Ferulic acid has been identified to be an active principle because it may reverse memory deficits induced by a variety of toxins, including dl-buthionine-(S,R)sulfoximine, trimethyltin, glutamate, $\mathrm{A} \beta 1-42$, scopolamine, and cycloheximide. Multiple mechanisms are probably implicated into its cognitive benefits, including inhibition on oxidative stress, activation of ChAT or enhance the cholinergic activities, competitive $\mathrm{N}$-methyl-D-aspartate (NMDA) receptor antagonism, suppression on immunoreactivities of the astrocyte, and facilitation of cerebral blood flow ([66-70], Table 6(b), Figure 3).

(2) Z-ligustilide has been identified to be another active component from volatile of Radix Angelica sinensis. It may protect brain and cognition especially against focal and global ischemia induced by permanent common carotid arteries occlusion (CCAO) and transient middle cerebral artery occlusion (MCAO) [71-73], (Table 6(b), Figure 3).

(3) Additionally, N-methyl-beta-carboline-3-carboxamide, Coniferyl ferulate, and 11-angeloylsenkyunolide $\mathrm{F}$ were identified to be anti-AD components probably by inhibiting $A \beta 1-40$ induced toxicity and $\mathrm{AChE}$ activity $([62,74]$, Figure 3$)$.

3.5. Radix rehmanniae. Radix rehmanniae is the roots of Rehmannia glutinosa Libosch., family Scrophulariaceae.

3.5.1. Functionality/Efficacy. There have been growing evidences that Radix rehmanniae extract possesses significant neuroprotective activity $([75,76]$, Table 7$)$. 
TABLE 5: Memory-improving and neuro-protective effects of Radix et rhizoma glycyrrhizae.

\begin{tabular}{|c|c|c|c|c|c|c|}
\hline Test & Test materials /dose & Test model & Endpoint/biomarkers & Effects & Mechanisms & Reference \\
\hline \multirow{6}{*}{ In vivo } & \multirow{3}{*}{$\begin{array}{l}\text { Extracts } \\
75-300 \mathrm{mg} / \mathrm{kg}, 7 \mathrm{~d} \\
\text { diet } \\
0.5 \text { or } 1 \%, 6 \mathrm{w}\end{array}$} & Diazepam treated mice & Elevated plus-maze test & \multirow{2}{*}{ Improve memory } & \multirow{2}{*}{ Cholinergic } & {$[50]$} \\
\hline & & Scopolamine treated mice & passive avoidance test & & & {$[51]$} \\
\hline & & $\mathrm{A} \beta 25-35$ treated mice & $\begin{array}{l}\text { passive avoidance test } \\
\text { Morris water-maze test } \\
\text { TBARS/Catalase/AChE }\end{array}$ & Improve memory & $\begin{array}{l}\text { Quench oxidative stress } \\
\text { Inhibit AChE }\end{array}$ & {$[52]$} \\
\hline & $\begin{array}{l}\text { Aqueous extracts } \\
150 \mathrm{mg} / \mathrm{kg}, 7 \mathrm{~d} \\
\mathrm{n} \text {-hexane extracts } \\
5 \mathrm{mg} / \mathrm{kg}, 3 \mathrm{~d}\end{array}$ & Innate mice & $\mathrm{AChE}$ & Inhibit AChE & N.A. & {$[53]$} \\
\hline & $\begin{array}{l}\text { Methanol extract } \\
50-100 \mathrm{mg} / \mathrm{kg}, 21 \mathrm{~d}\end{array}$ & $\begin{array}{l}\text { IR treated Mongolian } \\
\text { gerbils }\end{array}$ & $\begin{array}{l}\mathrm{Cu}, \mathrm{Zn}-\mathrm{SOD} 1 \\
\mathrm{CA} 1 \text { pyramidal cells }\end{array}$ & Protect neurons & Restore $\mathrm{Cu}, \mathrm{Zn}$-SOD1 & {$[54]$} \\
\hline & $\begin{array}{l}\text { Liquiritigenin } \\
2.3-21 \mathrm{mg} / \mathrm{kg}, 7 \mathrm{~d}\end{array}$ & $\mathrm{~A} \beta(25-35)$-treated rats & $\begin{array}{l}\text { Morris water maze test } \\
\text { Reference memory task } \\
\text { Probe task } \\
\text { Two-way shuttle } \\
\text { avoidance task } \\
\text { MAP, Nissle, Notch-2 }\end{array}$ & & & {$[55]$} \\
\hline \multirow{3}{*}{ In vivo } & $\begin{array}{l}\text { Isoliquiritigenin } \\
5-20 \mathrm{mg} / \mathrm{kg}, 7 \mathrm{~d}\end{array}$ & MCAO-treated rats & $\begin{array}{l}\text { MDA } \\
\text { SOD,GSH-Px, Catalase } \\
\mathrm{Na}^{+}-\mathrm{K}^{+}-\mathrm{ATPase} \text {, ATP } \\
\text { Energy charge, total } \\
\text { adenine nucleotides }\end{array}$ & Protect brain & $\begin{array}{l}\text { Promote energy } \\
\text { metabolism } \\
\text { Inhibit oxidative stress }\end{array}$ & {$[56]$} \\
\hline & $\begin{array}{l}\text { Glabridin } \\
1-4 \mathrm{mg} / \mathrm{kg}, 3 \mathrm{~d}\end{array}$ & Innate Mice & ChE & Improve memory & Inhibit ChE & {$[57]$} \\
\hline & $\begin{array}{l}\text { Glabridin } \\
5-25 \mathrm{mg} / \mathrm{kg}\end{array}$ & $\begin{array}{l}\text { IR-treated rats } \\
\text { Staurosporine-treated } \\
\text { neurons }\end{array}$ & $\begin{array}{l}\text { MDA, GSH and SOD } \\
\text { Bax, caspase-3,bcl-2 }\end{array}$ & Protect neurons & $\begin{array}{l}\text { Inhibit apoptosis } \\
\text { Inhibit oxidative stress }\end{array}$ & {$[58]$} \\
\hline
\end{tabular}

Acetylcholinesterase (AChE); cholinesterase (ChE); thiobarbituric acid-reactive substances (TBARS); superoxide dismutase (SOD); malondialdehyde (MDA); glutathione (GSH); microtubule-associated protein (MAP) 2; middle cerebral artery occlusion (MCAO); $\beta$ amyloid (A $\beta$ ); Ischemia-reperfusion (IR); not available (N.A.).<smiles>COc1cc(/C=C/C(=O)O)ccc1O</smiles>

(a)<smiles>CCC/C=C1\OC(=O)C2=C1CCC=C2</smiles>

Z-ligustilide

(b)<smiles>CNC(=O)c1cc2c(cn1)[nH]c1ccccc12</smiles>

$\mathrm{N}$-methyl-beta-carboline-3-carboxamide

(c)<smiles>COc1cc(/C=C/COC(=O)/C=C/c2ccc(O)c(OC)c2)ccc1O</smiles>

Coniferyl ferulate

(d)<smiles>C/C=C(/C)C(=O)OC(C=C1OC(=O)C2=C1CCC=C2)CC</smiles>

11-angeloylsenkyunolide $\mathrm{F}$

(e)

Figure 3: Chemical structures of ferulic acid, Z-ligustilide, N-methyl-beta-carboline-3-carboxamide, coniferyl ferulate, and 11angeloylsenkyunolide F. 
TABLE 6

(a) Memory-improving and neuro-protective effects of Radix Angelica sinensis

\begin{tabular}{|c|c|c|c|c|c|c|}
\hline Test & Test materials/dose & Test model & Endpoint/biomarkers & Effects & Mechanisms & Reference \\
\hline In vivo & $\begin{array}{l}\text { Extracts } \\
1 \mathrm{~g} / \mathrm{kg}\end{array}$ & $\begin{array}{l}\text { scopolamine-treated rats } \\
\text { cycloheximide-treated rats }\end{array}$ & Step-through test & Improve memory & N.A. & {$[62]$} \\
\hline In vitro & $\begin{array}{l}\text { Extracts } \\
25-200 \mu \mathrm{g} / \mathrm{ml}\end{array}$ & A $\beta$-treated Neuro 2A cells & $\begin{array}{l}\text { MTT assay/ } \Delta \Psi \mathrm{m} \\
\mathrm{ROS} / \mathrm{LPO} / \mathrm{GSH}\end{array}$ & Protect neurons & $\begin{array}{l}\text { Quench } \\
\text { oxidative stress }\end{array}$ & {$[63]$} \\
\hline
\end{tabular}

3-(4,5-dimethylthiazol-2yl)-2,5-diphenyltetrazolium bromide (MTT); Lipid peroxidation (LPO); mitochondrial transmembrane potential ( $\Delta \Psi \mathrm{m}) ; \beta$ amyloid $(\mathrm{A} \beta)$; glutathione $(\mathrm{GSH})$; not available (N.A.).

(b) Memory-improving and neuro-protective effects of active components from Radix Angelica sinensis

\begin{tabular}{|c|c|c|c|c|c|c|}
\hline Test & Test materials/dose & Test model & Endpoint/biomarkers & Effects & Mechanisms & Reference \\
\hline \multirow{8}{*}{ In vivo } & $\begin{array}{l}\text { Ferulic acid } \\
\text { s.c., } 5 \mathrm{mg} / \mathrm{kg} / \mathrm{d}, 6 \mathrm{~d}\end{array}$ & $\begin{array}{l}\text { dl-buthionine- }(\mathrm{S}, \mathrm{R})- \\
\text { Sulfoximine treated mice }\end{array}$ & $\begin{array}{l}\text { Object recognition test } \\
\text { Oxidative carbonyl } \\
\text { protein }\end{array}$ & Improve memory & $\begin{array}{l}\text { Elevate carbonyl } \\
\text { protein }\end{array}$ & {$[66]$} \\
\hline & $\begin{array}{l}\text { Ferulic acid } \\
28 \mathrm{~d}\end{array}$ & Trimethyltin-treated mice & $\begin{array}{l}\text { Y-maze test } \\
\text { Passive avoidance test } \\
\text { ChAT }\end{array}$ & Improve memory & Activate ChAT & {$[67]$} \\
\hline & $\begin{array}{l}\text { Ferulic acid } \\
\text { i.p., } 20-80 \mathrm{mg} / \mathrm{kg}, 3 \mathrm{~d}\end{array}$ & Glutamate-treated mice & $\begin{array}{l}\text { Behavioral test } \\
\text { histopathology } \\
{[(3) \mathrm{H}] \text {-labeled }} \\
\text { glutamate } \\
\text { bcl-2/caspase-3 }\end{array}$ & Protect brain & $\begin{array}{l}\text { NMDA receptor } \\
\text { antagonist }\end{array}$ & {$[68]$} \\
\hline & $\begin{array}{l}\text { Ferulic acid } \\
0.006 \%, 4 \mathrm{w}\end{array}$ & $\mathrm{A} \beta 1$-42-treated mice & $\begin{array}{l}\text { Step-through test } \\
\text { Y-maze test } \\
\text { Water maze test } \\
\text { GFAP/IL-1 } \beta\end{array}$ & $\begin{array}{l}\text { Improve memory } \\
\text { Protect brain }\end{array}$ & $\begin{array}{l}\text { Suppress astrocytes } \\
\text { immunoreactivities }\end{array}$ & {$[69]$} \\
\hline & $\begin{array}{l}\text { Ferulic acid } \\
50-100 \mathrm{mg} / \mathrm{kg}\end{array}$ & $\begin{array}{l}\text { Scopolamine-treated rats } \\
\text { Cycloheximide-treated } \\
\text { rats }\end{array}$ & Step-through test & Improve memory & $\begin{array}{l}\text { Cholinergic } \\
\text { Enhance CBF }\end{array}$ & {$[70]$} \\
\hline & $\begin{array}{l}\text { Z-ligustilide } \\
10-40 \mathrm{mg} / \mathrm{kg}, 4 \mathrm{w}\end{array}$ & CCAO-treated rats & $\begin{array}{l}\text { Morris water maze } \\
\text { Neurons/astrocytes } \\
\text { count } \\
\text { MDA/SOD/ChAT/AChE }\end{array}$ & Improve memory & $\begin{array}{l}\text { Inhibit oxidative } \\
\text { stress } \\
\text { Cholinergic }\end{array}$ & {$[71]$} \\
\hline & $\begin{array}{l}\text { Z-ligustilide } \\
20-80 \mathrm{mg} / \mathrm{kg}\end{array}$ & MCAO-treated rats & $\begin{array}{l}\text { TTC staining } \\
\text { Brain swelling } \\
\text { Behavioural score }\end{array}$ & Protect brain & N.A. & {$[72]$} \\
\hline & $\begin{array}{l}\text { Z-ligustilide } \\
5-20 \mathrm{mg} / \mathrm{kg}\end{array}$ & IR-treated ICR mice & $\begin{array}{l}\text { TTC staining } \\
\text { MDA/GSH-Px/SOD } \\
\text { Bcl-2/Bax/caspase-3 }\end{array}$ & Protect brain & $\begin{array}{l}\text { Inhibit oxidative } \\
\text { stress } \\
\text { Inhibit apoptosis }\end{array}$ & {$[73]$} \\
\hline
\end{tabular}

Choline acetyltransferase (ChAT); cerebral blood flow (CBF); glial fibrillary acidic protein (GFAP); interleukin-1 (IL-1); glutathione peroxidase (GSHPX); 2,3,5-triphenyltetrazolium chloride (TTC); subcutaneously (s.c.); ischemia-reperfusion (IR); superoxide dismutase (SOD); malondialdehyde (MDA); acetylcholinesterase (AChE); common carotid arteries occlusion (CCAO); middle cerebral artery occlusion (MCAO); $\beta$ amyloid (A $\beta$ ); N-methyl-D-aspartate (NMDA); not available (N.A.).

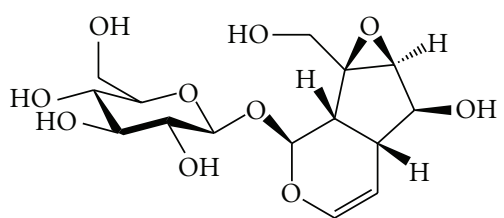

FIGURE 4: Chemical structure of catalpol.

3.5.2. Mechanism of Action. Radix rehmanniae extract improved learning and memory in rats with Monosodiumglutamate-(MSG-) injured thalamic arcuate nucleus at 4.5 , and $9.0 \mathrm{~g} / \mathrm{kg}$, through adjusting glutamates and $\gamma$ amiobutyic acid (GABA) levels, as well as increasing the expression of hippocampal c-fos, nerve growth factor (NGF), NMDA receptor 1, and GABA receptor. Moreover, Rehmannia extract stimulated glial cell-derived neurotrophic factor (GDNF) gene expression in C6 glioblastoma cells, through upregulating cPKC and ERK $1 / 2$ pathways ([76, 77], Table 7).

3.5.3. Active Components. Catalpol, an iridoid glycoside, was isolated from the fresh Radix rehmanniae. It exists broadly in many plants all over the world and has many biological functions such as anti-inflammation, promoting of sex hormones production, protection of liver damage, and reduction of elevated blood sugar. 
TABLE 7: Memory-improving and neuro-protective effects of Radix rehmanniae.

\begin{tabular}{|c|c|c|c|c|c|c|}
\hline Test & Test materials/dose & Test model & Endpoint/biomarkers & Effects & Mechanisms & Reference \\
\hline \multirow[b]{2}{*}{ In vivo } & $\begin{array}{l}\text { Extracts } \\
4.5-9.0 \mathrm{~g} / \mathrm{kg}\end{array}$ & MSG-treated rats & $\begin{array}{l}\text { Morris maze test } \\
\text { Step-down test } \\
\text { c-fos, NGF expression }\end{array}$ & Improve memory & $\begin{array}{l}\text { Motivate hippocampal } \\
\text { c-fos /NGF expression }\end{array}$ & {$[75]$} \\
\hline & $\begin{array}{l}\text { Extracts } \\
4.5-9.0 \mathrm{~g} / \mathrm{kg}\end{array}$ & MSG-treated rats & $\begin{array}{l}\text { Morris maze test } \\
\text { Step-down test } \\
\text { NMDA-R1, GABA-R } \\
\text { Glutamine, GABA } \\
\text { levels }\end{array}$ & Improve memory & $\begin{array}{l}\text { Motivate hippocampal } \\
\text { NMDA-R1/GABA-R } \\
\text { expression } \\
\text { adjust Glutamine/GABA } \\
\text { levels }\end{array}$ & {$[77]$} \\
\hline In vitro & $\begin{array}{l}\text { Extracts } \\
0.1-1.0 \mathrm{mg} / \mathrm{mL}, 1-3 \mathrm{~d}\end{array}$ & C6 glioblastoma cells & GDNF gene expression & $\begin{array}{l}\text { Stimulate GDNF } \\
\text { expression }\end{array}$ & $\begin{array}{l}\text { Up-regulate } \\
\text { cPKC/ERK1/2 pathways }\end{array}$ & {$[76]$} \\
\hline \multirow{4}{*}{ In vivo } & $\begin{array}{l}\text { Catalpol } \\
\text { i.p., } 10 \mathrm{mg} / \mathrm{kg}, 10 \mathrm{~d}\end{array}$ & LPS-treated mice & $\begin{array}{l}\text { MMP } \\
\text { NF- } \kappa \mathrm{B}\end{array}$ & $\begin{array}{l}\text { Improve memory } \\
\text { Inhibit inflammation }\end{array}$ & $\begin{array}{l}\text { Inhibit NF- } \kappa \mathrm{B} \text { activation } \\
\text { protect mitochondrial } \\
\text { function }\end{array}$ & {$[78]$} \\
\hline & $\begin{array}{l}\text { Catalpol } \\
2.5-10 \mathrm{mg} / \mathrm{kg}, 2 \mathrm{w}\end{array}$ & $\begin{array}{l}\text { D-galactose-treated } \\
\text { mice }\end{array}$ & $\begin{array}{l}\text { Passive avoidance test } \\
\text { LDH, GSH-ST, GS, CK }\end{array}$ & Improve memory & $\begin{array}{l}\text { Inhibit oxidative stress } \\
\text { Maintain energy } \\
\text { metabolism }\end{array}$ & {$[79-81]$} \\
\hline & $\begin{array}{l}\text { Catalpol } \\
\text { i.p., } 1-10 \mathrm{mg} / \mathrm{kg}\end{array}$ & IR-treated Gerbils & Bcl-2, Bax, NO & $\begin{array}{l}\text { Protect CA1 neurons } \\
\text { Improve memory }\end{array}$ & $\begin{array}{l}\text { Inhibit apoptosis } \\
\text { Inhibit oxidative stress }\end{array}$ & {$[82-84]$} \\
\hline & $\begin{array}{l}\text { Catalpol } \\
\text { i.p., } 5 \mathrm{mg} / \mathrm{kg}, 10 \mathrm{~d}\end{array}$ & Aged rats & $\begin{array}{l}\text { GAP-43/synaptophysin } \\
\text { PKC, BDNF }\end{array}$ & $\begin{array}{l}\text { Protect } \\
\text { neuroplasticity }\end{array}$ & $\begin{array}{l}\text { Up-regulate PKC and } \\
\text { BDNF (hippocampus) }\end{array}$ & {$[85]$} \\
\hline \multirow{7}{*}{ In vitro } & $\begin{array}{l}\text { Catalpol } \\
0.5 \mathrm{mM}, 1 \mathrm{~h}\end{array}$ & $\begin{array}{l}\text { MPTP-treated } \\
\text { neurons }\end{array}$ & $\begin{array}{l}\text { Cells Viability, } \\
\text { MAO-B, } \\
\text { ROS, MCI, MMP, MPT }\end{array}$ & Protect neurons & $\begin{array}{l}\text { Protect mitochondria } \\
\text { Maintain MAO-B } \\
\text { activity }\end{array}$ & {$[86]$} \\
\hline & $\begin{array}{l}\text { Catalpol } \\
0.5 \mathrm{mM}, 30 \mathrm{~min}\end{array}$ & $\begin{array}{l}\text { A } \beta 1-42 \text {-treated } \\
\text { Cortical neurons-glia }\end{array}$ & $\begin{array}{l}\text { Cells Viability } \\
\text { TNF- } \alpha \text {, iNOS, NO, } \\
\text { ROS }\end{array}$ & Protect neurons & Inhibit inflammation & {$[87]$} \\
\hline & $\begin{array}{l}\text { Catalpol } \\
0.25-5 \mathrm{mg} / \mathrm{ml}\end{array}$ & $\begin{array}{l}\text { Primary rat cortical } \\
\text { neurons }\end{array}$ & $\begin{array}{l}\text { Cells Viability } \\
\text { NF-200 antigen }\end{array}$ & $\begin{array}{l}\text { Enhance axonal } \\
\text { growth } \\
\text { No impact on survival }\end{array}$ & N.A. & {$[88]$} \\
\hline & $\begin{array}{l}\text { Catalpol } \\
0.1-100 \mu \mathrm{g} / \mathrm{ml}\end{array}$ & $\begin{array}{l}\text { OGD-treated PC12 } \\
\text { cells }\end{array}$ & $\begin{array}{l}\text { Bcl-2, caspase-3/MMP } \\
\text { SOD, GSH-Px }\end{array}$ & Inhibit apoptosis & $\begin{array}{l}\text { Retain Bcl-2 and MMP } \\
\text { suppress caspase-3 } \\
\text { activation } \\
\text { maintain SOD and } \\
\text { GSH-Px }\end{array}$ & [89] \\
\hline & $\begin{array}{l}\text { Catalpol } \\
0.1-1.0 \mathrm{mM}\end{array}$ & $\begin{array}{l}\mathrm{H}_{2} \mathrm{O}_{2} \text {-treated PC12 } \\
\text { cells }\end{array}$ & $\begin{array}{l}\text { Bcl-2 } \\
\text { cytochrome c caspase }\end{array}$ & $\begin{array}{l}\text { Protect neurons } \\
\text { Inhibit apoptosis }\end{array}$ & $\begin{array}{l}\text { Prevent cytochrome c } \\
\text { release } \\
\text { Inactivate caspase } \\
\text { cascade }\end{array}$ & {$[90]$} \\
\hline & $\begin{array}{l}\text { Catalpol } \\
0.05-0.5 \mathrm{mM}\end{array}$ & $\begin{array}{l}\mathrm{H}_{2} \mathrm{O}_{2} \text {-treated } \\
\text { astrocytes }\end{array}$ & $\begin{array}{l}\text { Cells Viability } \\
\text { ROS }\end{array}$ & Inhibit oxidative stress & $\begin{array}{l}\text { maintain glutathione } \\
\text { Scavenge ROS }\end{array}$ & {$[91]$} \\
\hline & $\begin{array}{l}\text { Catalpol } \\
0.3-275.9 \mu \mathrm{M}, 24 \mathrm{~h}\end{array}$ & $\begin{array}{l}\text { OGD-treated mice } \\
\text { astrocytes }\end{array}$ & $\begin{array}{l}\text { Cell survival/MMP } \\
\text { ROS, NO, iNOS, MDA } \\
\text { SOD, GSH-Px, GSH }\end{array}$ & Protect astrocytes & Inhibit oxidative stress & {$[92]$} \\
\hline
\end{tabular}

Nerve growth factor (NGF); oxygen-glucose deprivation (OGD); lactate dehydrogenase (LDH); glutathione S-transferase (GSH-ST); glutamine synthetase (GS); creatine kinase (CK); mitochondrial complex I (MCI); mitochondrial membrain potential (MMP); mitochondrial permeability transition (MPT); brain-derived neurotrophic factor (BDNF); $\gamma$-amiobutyic acid (GABA); lactate dehydrogenase (LDH); nitric oxide (NO); inducible nitric oxide synthase (iNOS); nuclear factor-kappa B (NF- $\kappa$ B); protein kinase C (PKC); 1-methyl-4-phenyl-1,2,3,6-tetrahydropyridine (MPTP); monosodium glutamate (MSG); lipopolysaccharide (LPS); ischemia-reperfusion (IR); monoamine oxidase (MAO); tumour necrosis factor (TNF)- $\alpha$; reactive oxygen species (ROS); superoxide dismutase (SOD); malondialdehyde (MDA); glutathione (GSH); glutathione peroxidase (GSH-Px); glial cell-derived neurotrophic factor (GDNF).

Recently, catalpol has been identified as a vital active with robust cognitive potential (Figure 4). Behaviour studies exhibited that catalpol reversed brain damage and memory deficits in mice induced by lipopolysaccharide (LPS) and D-galactose and in gerbils by cerebral ischemia. The nootropic and neuroprotective efficacy of catalpol probably resulted from a variety of underlying molecular mechanisms (Table 7).

(i) Antioxidant activity: catalpol promoted endogenous antioxidant enzyme activities, superoxide dismutase (SOD) and glutathione peroxidase (GSH-Px), and 
antioxidant glutathione (GSH), cut down malondialdehyde (MDA) and reactive oxygen species (ROS) generation in PC12 cells and astrocytes primary cultures, exposed to oxygen and glucose deprivation or $\mathrm{H}_{2} \mathrm{O}_{2}$, and in senescent mice induced by $\mathrm{D}$ galactose [79-81, 86, 89, 91, 92].

(ii) Anti-inflammatory activity: catalpol significantly reduced the release of ROS, TNF- $\alpha$, nitric oxide (NO) and inducible nitric oxide synthase (iNOS) expression after $\mathrm{A} \beta$ (1-42)-induced microglial activation in primary cortical neuron-glia cultures, and LPSinduced nuclear factor-kappa $\mathrm{B}(\mathrm{NF}-\kappa \mathrm{B})$ activation in mice $[78,87]$.

(iii) Neurogenetic activity: catalpol can enhance axonal growth of cortical neurons cultured in vitro from $24 \mathrm{~h}$ newly born rat, at $1-5 \mathrm{mg} / \mathrm{mL}$ and ameliorate agerelated presynaptic proteins decline (synaptophysin and GAP-43), and neuroplasticity loss in the hippocampus of the aged rats, by upregulating protein kinase $\mathrm{C}(\mathrm{PKC})$ and brain-derived neurotrophic factor (BDNF) [85, 88].

(iv) Antiapoptotic activity: catalpol not only suppressed the downregulation of $\mathrm{Bcl}-2$, upregulation of $\mathrm{Bax}$, and the release of mitochondrial cytochrome $\mathrm{c}$ to cytosol, but also attenuated caspase- 3 activation, poly-ADP-ribose polymerase (PARP) cleavage, and eventually protected against $\mathrm{H}_{2} \mathrm{O}_{2}$-induced apoptosis in PC12 cells and in the ischemic dorsal hippocampus of gerbils subject to CCAO [82-84, 90].

(v) In addition, the function to stimulate the production of adrenal cortical hormones, which increases the production of sex hormones, is likely implicated into the cognitive benefit of catalpol in menopausal women [92].

\section{Discussion and Conclusion}

TCM has a long history of human use for mental health. The current literature survey addressing traditional evidence from human studies has been primarily carried out. The top 10 TCM herb ingredients were identified. Poria, thinleaf milkwort, licorice, Chinese Angelica, and Rehmannia were further prioritized to have the highest potential benefit to dementia intervention, due to their highest frequency of use in 236 formulae collected from 29 ancient Pharmacopoeias, ancient formula books, or historical archives on ancient renowned TCM doctors, over the past 10 centuries.

In TCM philosophy, $\mathrm{AD}$ is assumed to be induced by kidney essence vacuity and toxin (turbid phlegm). The amnestic mild cognitive impairment in elderly population has been disclosed in a clinical investigation to correlate with kidney essence vacuity and turbid phlegm blocking upper orifices. The whole cognitive function may worsen because of the aggravation of kidney essence vacuity, deficiency of blood and qi, phlegm and heat toxin and may eventually lead to multiple cognitive domains impairment, even dementia [93].
Based on the history of use, there is strong clinical support that Radix polygalae is memory improving since its efficacy has been demonstrated in elderly with mild cognitive decline $[32,33]$. There is suggestive evidence that Poria cocos, Radix glycyrrhizae, Radix Angelica sinensis, or Radix rehmanniae are memory improving, though modern clinical reports concerning the four herbs are absent yet.

Furthermore, pharmacological investigations in 39 animal studies and 18 in vitro studies also indicated that the five ingredients can elicit memory-improving effects via multiple mechanisms of action, covering estrogen-like, cholinergic, antioxidant, anti-inflammatory, antiapoptotic, Neurogenetic, and anti-A $\beta$ activities. These mechanisms are in well accordance with modern pharmacotherapy for $\mathrm{AD}$ and VD, by prescribing ChEIs, anti-inflammatory mediations, antioxidants, estrogen, neurotrophic factors, and nootropics, depending on difference situations.

In the meantime, 11 active molecules have also been identified, including sinapic acid, tenuifolin, isoliquiritigenin, liquiritigenin, glabridin, ferulic acid, Z-ligustilide, N-methyl-beta-carboline-3-carboxamide, coniferyl ferulate and 11-angeloylsenkyunolide F, and catalpol. Most of them are lipophilic compounds with comparatively low-molecular weight $(200 \sim 700)$ and likely to be absorbed into blood and distributed to brain according to Lipinski rule of 5 [94]. The 11 compounds can serve as active markers for characterisation and standardization of corresponding TCM herbal extracts and pharmacokinetics markers for bioavailability study. In drug discovery, these phyto-chemicals can also be used as candidates to optimize derivatives [95].

Taken together, it is concluded that TCM could have a complementary and alternative role in preventing and treating cognitive disorder in the elderly. The scientific evidence is being continuously mined to back up the traditional medical wisdom and product innovation in the healthcare sectors.

\section{Acknowledgments}

The authors thank Dr. Domenic Caravetta from Unilever R\&D Shanghai and Dr. Jan Koek from Unilever R\&D Vlaardingen for reviewing the paper.

\section{References}

[1] E. L. Nestler, S. E. Hyman, and R. C. Malenka, Molecular Neuropharmacology: A Foundation for Clinical Neuroscience, McGraw-Hill, New York, NY, USA, 2001.

[2] D. I. Dominguez and B. D. Strooper, "Novel therapeutic strategies provide the real test for the amyloid hypothesis of Alzheimer's disease," Trends in Pharmacological Sciences, vol. 23, no. 7, pp. 324-330, 2002.

[3] H. Sugimoto, "The new approach in development of antiAlzheimer's disease drugs via the cholinergic hypothesis," Chemico-Biological Interactions, vol. 175, no. 1-3, pp. 204-208, 2008.

[4] L. E. Rojo, J. A. Fernández, A. A. Maccioni, J. M. Jimenez, and R. B. Maccioni, "Neuroinflammation: implications for the pathogenesis and molecular diagnosis of Alzheimer's disease," Archives of Medical Research, vol. 39, no. 1, pp. 1-16, 2008. 
[5] D. Praticò and J. Q. Trojanowski, "Inflammatory hypotheses: novel mechanisms of Alzheimer's neurodegeneration and new therapeutic targets?" Neurobiology of Aging, vol. 21, no. 3, pp. 441-445, 2000.

[6] W. R. Markesbery, “Oxidative stress hypothesis in Alzheimer's disease," Free Radical Biology and Medicine, vol. 23, no. 1, pp. 134-147, 1997.

[7] A. Savioz, G. Leuba, P. G. Vallet, and C. Walzer, "Contribution of neural networks to Alzheimer disease's progression," Brain Research Bulletin, vol. 80, no. 4-5, pp. 309-314, 2009.

[8] C. E. Gleason, B. Cholerton, C. M. Carlsson, S. C. Johnson, and S. Asthana, "Neuroprotective effects of female sex steroids in humans: current controversies and future directions," Cellular and Molecular Life Sciences, vol. 62, no. 3, pp. 299312, 2005.

[9] C. S. Atwood and R. L. Bowen, "The reproductive-cell cycle theory of aging: an update," Experimental Gerontology, vol. 46, no. 2-3, pp. 100-107, 2010.

[10] A. Ott, M. M. B. Breteler, F. Van Harskamp et al., "Prevalence of Alzheimer's disease and vascular dementia: association with education. The Rotterdam study," British Medical Journal, vol. 310, no. 6985, pp. 970-973, 1995.

[11] K. Ritchie and S. Lovestone, "The dementias," Lancet, vol. 360, no. 9347, pp. 1759-1766, 2002.

[12] M. S. Parihar and T. Hemnani, "Alzheimer's disease pathogenesis and therapeutic interventions," Journal of Clinical Neuroscience, vol. 11, no. 5, pp. 456-467, 2004.

[13] C. N. Johnson, A. Roland, and N. Upton, "New symptomatic strategies in Alzheimer's disease," Drug Discovery Today: Therapeutic Strategies, vol. 1, no. 1, pp. 13-19, 2004.

[14] M. Adams, F. Gmünder, and M. Hamburger, "Plants traditionally used in age related brain disorders-a survey of ethnobotanical literature," Journal of Ethnopharmacology, vol. 113, no. 3, pp. 363-381, 2007.

[15] T. S. Anekonda and P. H. Reddy, "Can herbs provide a new generation of drugs for treating Alzheimer's disease?" Brain Research Reviews, vol. 50, no. 2, pp. 361-376, 2005.

[16] M. J. Howes and P. J. Houghton, "Plants used in Chinese and Indian traditional medicine for improvement of memory and cognitive function," Pharmacology Biochemistry and Behavior, vol. 75, no. 3, pp. 513-527, 2003.

[17] X. Liu, J. Du, J. Cai et al., "Clinical systematic observation of Kangxin capsule curing vascular dementia of senile kidney deficiency and blood stagnation type," Journal of Ethnopharmacology, vol. 112, no. 2, pp. 350-355, 2007.

[18] Chinese Pharmacopoeia Commission, Pharmacopoeia of the People's Republic of China (2005), vol. 1, People's Medical Publishing House, Beijing, China, 2005.

[19] L. Yan, B. Liu, W. Guo et al., "A clinical investigation on zhi ling tang for treatment of senile dementia," Journal of Traditional Chinese Medicine, vol. 20, no. 2, pp. 83-86, 2000.

[20] I. Hatip-Al-Khatib, N. Egashira, K. Mishima et al., "Determination of the effectiveness of components of the herbal medicine Toki-Shakuyaku-San and fractions of Angelica acutiloba in improving the scopolamine-induced impairment of rat's spatial cognition in eight-armed radial maze test," Journal of Pharmacological Sciences, vol. 96, no. 1, pp. 33-41, 2004.

[21] M. Smriga, H. Saito, and N. Nishiyama, "Hoelen (Poria cocos WOLF) and ginseng (Panax Ginseng C.A. MEYER), the ingredients of a chinese prescription DX-9386, individually promote hippocampal long-term potentiation in vivo," Biological and Pharmaceutical Bulletin, vol. 18, no. 4, pp. 518-522, 1995.
[22] M. H. Oh, P. J. Houghton, W. K. Whang, and J. H. Cho, "Screening of Korean herbal medicines used to improve cognitive function for anti-cholinesterase activity," Phytomedicine, vol. 11, no. 6, pp. 544-548, 2004.

[23] Z. Lin, Z. Xiao, D. Zhu, Y. Yan, B. Yu, and Q. Wang, "Aqueous extracts of FBD, a Chinese herb formula composed of Poria cocos, Atractylodes macrocephala, and Angelica sinensis reverse scopolamine induced memory deficit in ICR mice," Pharmaceutical Biology, vol. 47, no. 5, pp. 396-401, 2009.

[24] W. Chen, W. An, and J. Chu, "Effect of water extract of Poria on cytosolic free calcium concentration in brain nerve cells of neonatal rats," Zhongguo Zhong Xi Yi Jie He Za Zhi, vol. 18, no. 5, pp. 293-295, 1998.

[25] Q. Huang, Y. Jin, L. Zhang, P. C. K. Cheung, and J. F. Kennedy, "Structure, molecular size and antitumor activities of polysaccharides from Poria cocos mycelia produced in fermenter," Carbohydrate Polymers, vol. 70, no. 3, pp. 324-333, 2007.

[26] H. M. Kang, S. K. Lee, D. S. Shin et al., "Dehydrotrametenolic acid selectively inhibits the growth of H-ras transformed rat2 cells and induces apoptosis through caspase-3 pathway," Life Sciences, vol. 78, no. 6, pp. 607-613, 2006.

[27] S. J. Wu, L. T. Ng, and C. C. Lin, "Antioxidant activities of some common ingredients of traditional chinese medicine, Angelica sinensis, Lycium barbarum and Poria cocos," Phytotherapy Research, vol. 18, no. 12, pp. 1008-1012, 2004.

[28] N. Sekiya, H. Goto, Y. Shimada, Y. Endo, I. Sakakibara, and K. Terasawa, "Inhibitory effects of triterpenes isolated from Hoelen on free radical-induced lysis of red blood cells," Phytotherapy Research, vol. 17, no. 2, pp. 160-162, 2003.

[29] Z. Lin, D. Zhu, Y. Yan, and B. Yu, "Herbal formula FBD extracts prevented brain injury and inflammation induced by cerebral ischemia-reperfusion," Journal of Ethnopharmacology, vol. 118, no. 1, pp. 140-147, 2008.

[30] H. Chang and P. P. But, Pharmacology and Applications of Chinese Material Medica, vol. 1-2, World Scientific, Singapore, 2001.

[31] J. A. Duke and E. S. Ayensu, Medicinal Plants of China, vol. 12, Reference Publications, Algonac, Mich, USA, 2nd edition, 1985.

[32] H. Jung, J. Y. Lee, S. H. Kim, K. Y. Shin, G. H. Lee, and Y. H. Suh, "Effect of functional diet Polygala tenuifolia Willdenow extract [BT-11] on memory function of healthy general population," European Neuropsychopharmacology, vol. 17, no. 9, p. S526, 2007.

[33] S. H. Kim, H. Y. Jung, J. S. Yi et al., "Effect of functional diet BT-11 [Polygala tenuifolia Willdenow] on subjective memory impairment and mild cognitive impairment in older people," European Neuropsychopharmacology, vol. 16, p. S477, 2006.

[34] H. J. Park, K. Lee, H. Heo et al., "Effects of Polygala tenuifolia root extract on proliferation of neural stem cells in the hippocampal CA1 region," Phytotherapy Research, vol. 22, no. 10, pp. 1324-1329, 2008.

[35] K. Y. Shin, B. Y. Won, C. Heo et al., "BT-11 improves stressinduced memory impairments through increment of glucose utilization and total neural cell adhesion molecule levels in rat brains," Journal of Neuroscience Research, vol. 87, no. 1, pp. 260-268, 2009.

[36] Y. L. Chen, C. L. Hsieh, P. H. B. Wu, and J. G. Lin, "Effect of Polygala tenuifolia root on behavioral disorders by lesioning nucleus basalis magnocellularis in rat," Journal of Ethnopharmacology, vol. 95, no. 1, pp. 47-55, 2004. 
[37] C. H. Park, S. H. Choi, J. W. Koo et al., "Novel cognitive improving and neuroprotective activities of Polygala tenuifolia willdenow extract, BT-11," Journal of Neuroscience Research, vol. 70, no. 3, pp. 484-492, 2002.

[38] H. J. Lee, J. Y. Ban, S. B. Koh et al., "Polygalae radix extract protects cultured rat granule cells against damage induced by NMDA," American Journal of Chinese Medicine, vol. 32, no. 4, pp. 599-610, 2004.

[39] R. Naito and C. Tohda, "Characterization of anti-neurodegenerative effects of Polygala tenuifolia in A $\beta$ (25-35)-treated cortical neurons," Biological and Pharmaceutical Bulletin, vol. 29, no. 9, pp. 1892-1896, 2006.

[40] H. M. Kim, E. H. Lee, H. J. Na et al., "Effect of Polygala tenuifolia root extract on the tumor necrosis factor- $\alpha$ secretion from mouse astrocytes," Journal of Ethnopharmacology, vol. 61, no. 3, pp. 201-208, 1998.

[41] H. N. Koo, H. J. Jeong, K. R. Kim et al., "Inhibitory effect of interleukin- $1 \alpha$-induced apoptosis by Polygala tenuifolia in Hep H2 cells," Immunopharmacology and Immunotoxicology, vol. 22, pp. 531-544, 2000.

[42] X. L. Sun, H. Ito, T. Masuoka, C. Kamei, and T. Hatano, "Effect of Polygala tenuifolia root extract on scopolamine-induced impairment of rat spatial cognition in an eight-arm radial maze task," Biological and Pharmaceutical Bulletin, vol. 30, no. 9, pp. 1727-1731, 2007.

[43] T. Yabe, S. Iizuka, Y. Komatsu, and H. Yamada, "Enhancements of choline acetyltransferase activity and nerve growth factor secretion by Polygalae radix-extract containing active ingredients in Kami-untan-to," Phytomedicine, vol. 4, no. 3, pp. 199205, 1997.

[44] Y. Ikeya, S. Takeda, M. Tunakawa et al., "Cognitive improving and cerebral protective effects of acylated oligosaccharides in Polygala tenuifolia," Biological and Pharmaceutical Bulletin, vol. 27, no. 7, pp. 1081-1085, 2004.

[45] H. Zhang, T. Han, L. Zhang et al., "Effects of tenuifolin extracted from radix polygalae on learning and memory: a behavioral and biochemical study on aged and amnesic mice," Phytomedicine, vol. 15, no. 8, pp. 587-594, 2008.

[46] Q. Chen, Y. G. Cao, and C. H. Zhang, "Effect of tenuigenin on cholinergic decline induced by $\beta$-amyloid peptide and ibotenic acid in rats," Acta Pharmaceutica Sinica, vol. 37, no. 12, pp. 913-917, 2002.

[47] H. Jia, Y. Jiang, Y. Ruan et al., “Tenuigenin treatment decreases secretion of the Alzheimer's disease amyloid $\beta$-protein in cultured cells," Neuroscience Letters, vol. 367, no. 1, pp. 123128, 2004.

[48] C. Li, J. Yang, S. Yu et al., "Triterpenoid saponins with neuroprotective effects from the roots of Polygala tenuifolia," Planta Medica, vol. 74, no. 2, pp. 133-141, 2008.

[49] F. Karakida, Y. Ikeya, M. Tsunakawa et al., "Cerebral protective and cognition-improving effects of sinapic acid in rodents," Biological and Pharmaceutical Bulletin, vol. 30, no. 3, pp. 514519, 2007.

[50] D. Dhingra, M. Parle, and S. K. Kulkarni, "Memory enhancing activity of Glycyrrhiza glabra in mice," Journal of Ethnopharmacology, vol. 91, no. 2-3, pp. 361-365, 2004.

[51] M. Parle, D. Dhingra, and S. K. Kulkarni, "Memorystrengthening activity of Glycyrrhiza glabra in exteroceptive and interoceptive behavioral models," Journal of Medicinal Food, vol. 7, no. 4, pp. 462-466, 2004.

[52] J. Ahn, M. Um, W. Choi, S. Kim, and T. Ha, "Protective effects of Glycyrrhiza uralensis Fisch. on the cognitive deficits caused by $\beta$-amyloid peptide $25-35$ in young mice," Biogerontology, vol. 7, no. 4, pp. 239-247, 2006.
[53] D. Dhingra, M. Parle, and S. K. Kulkarni, "Comparative brain cholinesterase-inhibiting activity of Glycyrrhiza glabra, Myristica fragrans, ascorbic acid, metrifonate in mice," Journal of Medicinal Food, vol. 9, no. 2, pp. 281-283, 2006.

[54] I. K. Hwang, S. S. Lim, K. H. Choi et al., "Neuroprotective effects of roasted licorice, not raw form, on neuronal injury in gerbil hippocampus after transient forebrain ischemia," Acta Pharmacologica Sinica, vol. 27, no. 8, pp. 959-965, 2006.

[55] R. T. Liu, L. B. Zou, J. Y. Fu, and Q. J. Lu, "Effects of liquiritigenin treatment on the learning and memory deficits induced by amyloid $\beta$-peptide (25-35) in rats," Behavioural Brain Research, vol. 210, no. 1, pp. 24-31, 2010.

[56] C. Zhan and J. Yang, "Protective effects of isoliquiritigenin in transient middle cerebral artery occlusion-induced focal cerebral ischemia in rats," Pharmacological Research, vol. 53, no. 3, pp. 303-309, 2006.

[57] Y. M. Cui, M. Z. Ao, W. Li, and L. J. Yu, "Effect of glabridin from Glycyrrhiza glabra on learning and memory in mice," Planta Medica, vol. 74, no. 4, pp. 377-380, 2008.

[58] X. Q. Yu, C. C. Xue, Z. W. Zhou et al., "in vitro and in vivo neuroprotective effect and mechanisms of glabridin, a major active isoflavan from Glycyrrhiza glabra (licorice)," Life Sciences, vol. 82, no. 1-2, pp. 68-78, 2008.

[59] P. I. Hillerns, Y. Zu, Y. J. Fu, and M. Wink, "Binding of phytoestrogens to rat uterine estrogen receptors and human sex hormone-binding globulins," Zeitschrift fur Naturforschung, Section C, vol. 60, no. 7-8, pp. 649-656, 2005.

[60] S. Tamir, M. Eizenberg, D. Somjen, S. Izrael, and J. Vaya, "Estrogen-like activity of glabrene and other constituents isolated from licorice root," Journal of Steroid Biochemistry and Molecular Biology, vol. 78, no. 3, pp. 291-298, 2001.

[61] D. Somjen, E. Knoll, J. Vaya, N. Stern, and S. Tamir, "Estrogen-like activity of licorice root constituents: glabridin and glabrene, in vascular tissues in vitro and in vivo," Journal of Steroid Biochemistry and Molecular Biology, vol. 91, no. 3, pp. 147-155, 2004.

[62] M. T. Hsich, Y. T. Lin, Y. H. Lin, and C. R. Wu, "Radix Angelica sinensis extracts ameliorate scopolamine- and cycloheximideinduced amnesia, but not p-chloroamphetamine-induced amnesia in rats," American Journal of Chinese Medicine, vol. 28, no. 2, pp. 263-272, 2000.

[63] S. H. Huang, C. M. Lin, and B. H. Chiang, "Protective effects of Angelica sinensis extract on amyloid $\beta$-peptide-induced neurotoxicity," Phytomedicine, vol. 15, no. 9, pp. 710-721, 2008.

[64] C. Circosta, R. De Pasquale, D. R. Palumbo, S. Samperi, and F. Occhiuto, "Estrogenic activity of standardized extract of Angelica sinensis," Phytotherapy Research, vol. 20, no. 8, pp. 665-669, 2006.

[65] C. B. S. Lau, T. C. Y. Ho, T. W. L. Chan, and S. C. F. Kim, "Use of dong quai (Angelica sinensis) to treat peri- or postmenopausal symptoms in women with breast cancer: is it appropriate?" Menopause, vol. 12, no. 6, pp. 734-740, 2005.

[66] T. Mamiya, M. Kise, and K. Morikawa, "Ferulic acid attenuated cognitive deficits and increase in carbonyl proteins induced by buthionine-sulfoximine in mice," Neuroscience Letters, vol. 430, no. 2, pp. 115-118, 2008.

[67] M. J. Kim, S. J. Choi, S.-T. Lim et al., "Ferulic acid supplementation prevents trimethyltin-induced cognitive deficits in mice," Bioscience, Biotechnology and Biochemistry, vol. 71, no. 4, pp. 1063-1068, 2007.

[68] L. Yu, Y. Zhang, R. Ma, L. Bao, J. Fang, and T. Yu, "Potent protection of ferulic acid against excitotoxic effects of maternal intragastric administration of monosodium glutamate at a late 
stage of pregnancy on developing mouse fetal brain," European Neuropsychopharmacology, vol. 16, no. 3, pp. 170-177, 2006.

[69] J. J. Yan, J. Y. Cho, H. S. Kim et al., "Protection against $\beta$ amyloid peptide toxicity in vivo with long-term administration of ferulic acid," British Journal of Pharmacology, vol. 133, no. 1, pp. 89-96, 2001.

[70] M. T. Hsieh, F. H. Tsai, Y. C. Lin, W. H. Wang, and C. R. $\mathrm{Wu}$, "Effects of ferulic acid on the impairment of inhibitory avoidance performance in rats," Planta Medica, vol. 68, no. 8, pp. 754-756, 2002.

[71] X. Kuang, J. R. Du, Y. X. Liu, G. Y. Zhang, and H. Y. Peng, "Postischemic administration of Z-Ligustilide ameliorates cognitive dysfunction and brain damage induced by permanent forebrain ischemia in rats," Pharmacology Biochemistry and Behavior, vol. 88, no. 3, pp. 213-221, 2008.

[72] H. Y. Peng, J. R. Du, G. Y. Zhang et al., "Neuroprotective effect of Z-Ligustilide against permanent focal ischemic damage in rats," Biological and Pharmaceutical Bulletin, vol. 30, no. 2, pp. 309-312, 2007.

[73] X. Kuang, Y. Yao, J. R. Du, Y. X. Liu, C. Y. Wang, and Z. M. Qian, "Neuroprotective role of Z-ligustilide against forebrain ischemic injury in ICR mice," Brain Research, vol. 1102, no. 1, pp. 145-153, 2006.

[74] C. C. Ho, A. Kumaran, and L. S. Hwang, "Bio-assay guided isolation and identification of anti-Alzheimer active compounds from the root of Angelica sinensis," Food Chemistry, vol. 114, no. 1, pp. 246-252, 2009.

[75] Y. Cui, Z. Yan, S. Hou, and Z. Chang, "Effect of radix Rehmanniae preparata on the expression of c-fos and NGF in hippocampi and learning and memory in rats with damaged thalamic arcuate nucleus," Zhong Yao Cai, vol. 27, no. 8, pp. 589-592, 2004.

[76] H. Yu, K. Oh-Hashi, T. Tanaka et al., "Rehmannia glutinosa induces glial cell line-derived neurotrophic factor gene expression in astroglial cells via cPKC and ERK1/2 pathways independently," Pharmacological Research, vol. 54, no. 1, pp. 39-45, 2006.

[77] Y. Cui, Z. H. Yan, S. L. Hou, and Z. F. Chang, "Effect of Shu Dihuang on the transmitter and receptor of amino acid in brain and learning and memory of dementia model," Zhongguo Zhongyao Zazhi, vol. 28, no. 9, pp. 862-866, 2003.

[78] A. Zhang, S. Hao, J. Bi et al., "Effects of catalpol on mitochondrial function and working memory in mice after lipopolysaccharide-induced acute systemic inflammation," Experimental and Toxicologic Pathology, vol. 61, no. 5, pp. 461469, 2009.

[79] X. Zhang, A. Zhang, B. Jiang, Y. Bao, J. Wang, and L. An, "Further pharmacological evidence of the neuroprotective effect of catalpol from Rehmannia glutinosa," Phytomedicine, vol. 15, no. 6-7, pp. 484-490, 2008.

[80] X. L. Zhang, L. J. An, Y. M. Bao, J. Y. Wang, and B. Jiang, "d-galactose administration induces memory loss and energy metabolism disturbance in mice: protective effects of catalpol," Food and Chemical Toxicology, vol. 46, no. 8, pp. 2888-2894, 2008.

[81] X. L. Zhang, B. Jiang, Z. B. Li, S. Hao, and L. J. An, "Catalpol ameliorates cognition deficits and attenuates oxidative damage in the brain of senescent mice induced by d-galactose," Pharmacology Biochemistry and Behavior, vol. 88, no. 1, pp. 64-72, 2007.

[82] D. Q. Li, Y. L. Duan, Y. M. Bao, C. P. Liu, Y. Liu, and L. J. An, "Neuroprotection of catalpol in transient global ischemia in gerbils," Neuroscience Research, vol. 50, no. 2, pp. 169-177, 2004.
[83] D. Q. Li, Y. Li, Y. Liu, Y. M. Bao, B. Hu, and L. J. An, "Catalpol prevents the loss of CA1 hippocampal neurons and reduces working errors in gerbils after ischemia-reperfusion injury," Toxicon, vol. 46, no. 8, pp. 845-851, 2005.

[84] D. Q. Li, Y. M. Bao, Y. Li, C. F. Wang, Y. Liu, and L. J. An, "Catalpol modulates the expressions of Bcl-2 and Bax and attenuates apoptosis in gerbils after ischemic injury," Brain Research, vol. 1115, no. 1, pp. 179-185, 2006.

[85] J. Liu, Q. J. He, W. Zou et al., "Catalpol increases hippocampal neuroplasticity and up-regulates PKC and BDNF in the aged rats," Brain Research, vol. 1123, no. 1, pp. 68-79, 2006.

[86] J. Bi, X. B. Wang, L. Chen et al., "Catalpol protects mesencephalic neurons against MPTP induced neurotoxicity via attenuation of mitochondrial dysfunction and MAO-B activity," Toxicology in Vitro, vol. 22, no. 8, pp. 1883-1889, 2008.

[87] B. Jiang, J. Du, J. H. Liu, Y. M. Bao, and L. J. An, "Catalpol attenuates the neurotoxicity induced by $\beta$-amyloid (1-42) in cortical neuron-glia cultures," Brain Research, vol. 1188, no. 1, pp. 139-147, 2008.

[88] D. Wan, H. F. Zhu, Y. Luo, P. Xie, and X. Y. Xu, "Study of catalpol promoting axonal growth for cultured cortical neurons from rats," Zhongguo Zhongyao Zazhi, vol. 32, no. 17, pp. 1771-1774, 2007.

[89] Z. Wang, L. J. An, Y. L. Duan, Y. C. Li, and B. Jiang, "Catalpol protects rat pheochromocytoma cells against oxygen and glucose deprivation-induced injury," Neurological Research, vol. 30, no. 1, pp. 106-112, 2008.

[90] B. Jiang, J. H. Liu, Y. M. Bao, and L. J. An, "Catalpol inhibits apoptosis in hydrogen peroxide-induced PC12 cells by preventing cytochrome $\mathrm{c}$ release and inactivating of caspase cascade," Toxicon, vol. 43, no. 1, pp. 53-59, 2004.

[91] J. Bi, B. Jiang, J. H. Liu, C. Lei, X. L. Zhang, and L. J. An, "Protective effects of catalpol against $\mathrm{H}_{2} \mathrm{O}_{2}$-induced oxidative stress in astrocytes primary cultures," Neuroscience Letters, vol. 442, no. 3, pp. 224-227, 2008.

[92] Y. Li, Y. Bao, B. Jiang et al., "Catalpol protects primary cultured astrocytes from in vitro ischemia-induced damage," International Journal of Developmental Neuroscience, vol. 26, no. 3-4, pp. 309-317, 2008.

[93] Y. C. Miao, J. Z. Tian, J. Shi et al., "Correlation between cognitive functions and syndromes of traditional Chinese medicine in amnestic mild cognitive impairment," Zhong $X i$ Yi Jie He Xue Bao, vol. 7, no. 3, pp. 205-211, 2009.

[94] B. G. Giménez, M. S. Santos, M. Ferrarini, and J. P. Fernandes, "Evaluation of blockbuster drugs under the rule-of-five," Pharmazie, vol. 65, no. 2, pp. 148-152, 2010.

[95] A. Boultadakis, P. Liakos, and N. Pitsikas, "The nitric oxide-releasing derivative of ferulic acid NCX 2057 antagonized delay-dependent and scopolamine-induced performance deficits in a recognition memory task in the rat," Progress in Neuro-Psychopharmacology and Biological Psychiatry, vol. 34, no. 1, pp. 5-9, 2010. 


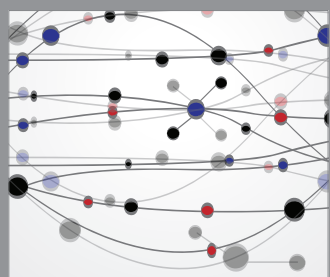

The Scientific World Journal
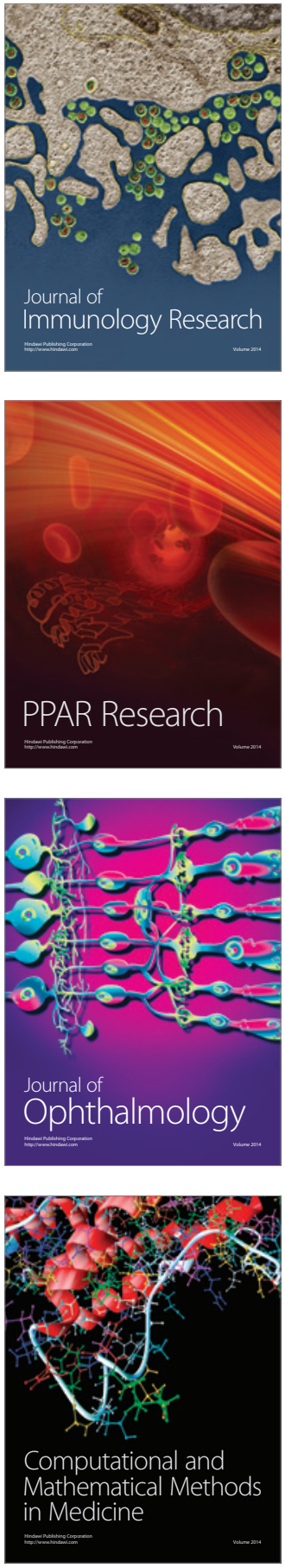

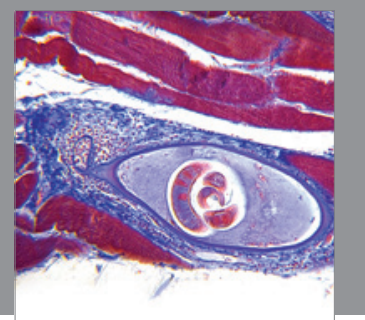

Gastroenterology

Research and Practice
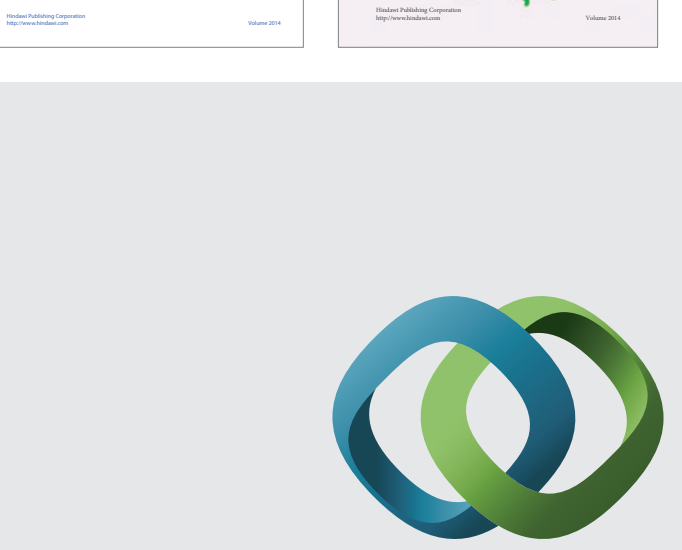

\section{Hindawi}

Submit your manuscripts at

http://www.hindawi.com
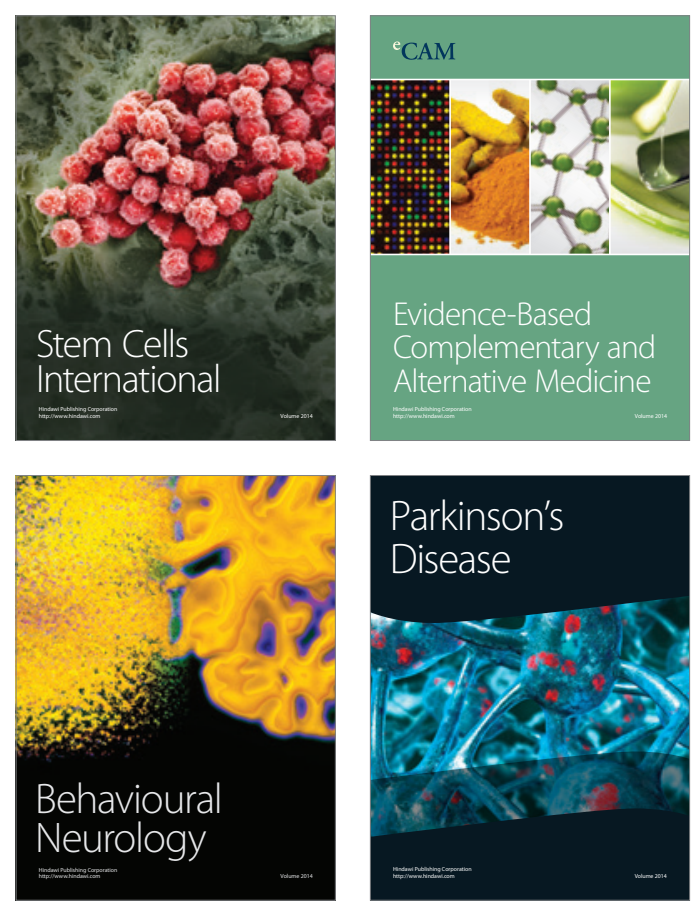

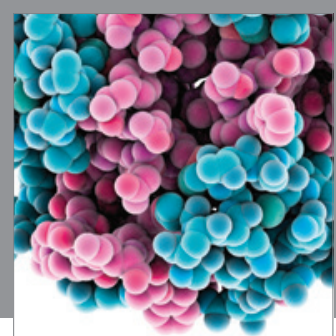

Journal of
Diabetes Research

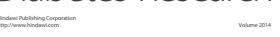

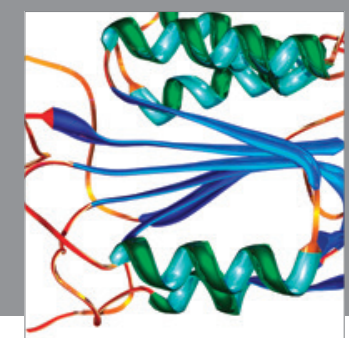

Disease Markers
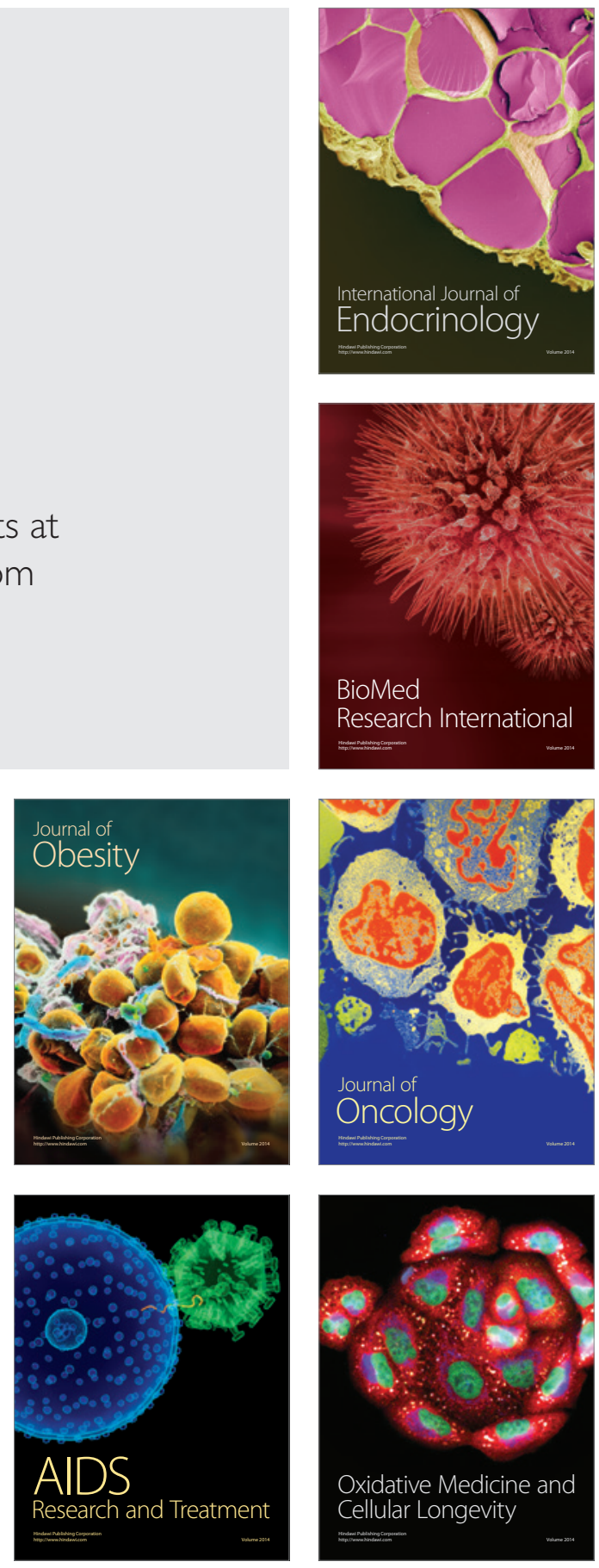